\title{
Triple-negative breast cancer - the past, present and future: recent and emerging trends in immunotherapy
}
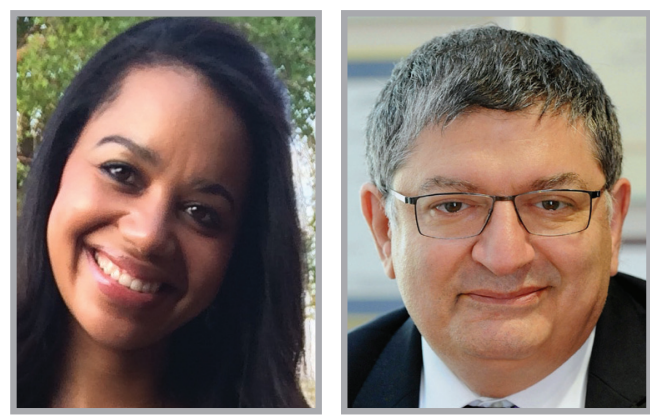

\author{
“Triple-negative breast cancer \\ is known to be far more \\ aggressive than other types of \\ breast cancers.”
}

Ronwyn van Eeden ${ }^{1} \&$ Bernardo L Rapoport ${ }^{*, 1}$

First draft submitted: 3 January 2016; Accepted for publication; 4 January 2016; Published online: 26 January 2016

There have been few significant advances in the treatment of triple-negative breast cancer (TNBC) until now. TNBC is characterized by its lack of expression of hormone receptors, ER and PR and lack of the HER2 receptor. In an attempt to better understand TNBC and to identify targets, it is known, although with controversy, that about $70 \%$ of these cancers have a basal-like profile on gene-expression analysis (defined by the expression of basal cytokeratins). The term is occasionally used to imply that all TNBCs are not alike, and that it may cause some TNBCs to behave differently clinically, or to have different prognostic indicators. Basal-like TNBC can encompass different histological subtypes comprising mostly medullary types, but also invasive ductal carcinomas of no special type, metaplastic types and sometimes lobular types, in addition to other subtypes. It is also shown in some studies that basal-like TNBC, which are in the majority, are often of high grade and have a high mitosis index with a worse outcome.

TNBC is known to be far more aggressive than other types of breast cancers. It has a quicker time to progression, as well as the ability to metastasize early in the disease process, particularly with visceral metastasis. These are all factors associated with shorter overall survival in patients.

TNBC represents $15-20 \%$ of all breast cancers. Clinicians are therefore often faced with difficult treatment decisions and fewer options than what is available for hormone-receptor positive and HER2positive disease. The management of TNBC remains an unmet medical need.

The mainstay of treatment is chemotherapy, namely anthracycline, taxane and more recently, platinum-based regimens. However, even though some tumors have higher response rates to chemotherapy, chemotherapy has limited efficacy in terms of survival, in both early and advanced stages of TNBC [1-3].

In the preoperative neoadjuvant setting, some trials have confirmed that TNBC has significantly higher pathological complete response (pCR) rates in comparison to hormone receptor-positive disease. In addition, various studies have shown that $\mathrm{pCR}$ correlates well

'The Medical Oncology Centre Rosebank, Johannesburg, South Africa

*Author for correspondence: brapoport@rosebankoncology.co.za

\section{KEYWORDS}

- antitumor vaccines • basal-like

- CTLA4 • IDO • immunotherapy

- interleukin therapy • PD-1 • PD-L1

- TIL • TNBC • triple-negative breast cancer

"Patients who attain a 5-year disease-free benchmark have a better outcome compared with those patients who relapse early in the disease course. In this clinical setting, recurrence or progression is unlikely to occur. Because triple-negative breast cancer has a poor prognosis, this 5-year benchmark is a significant goal to achieve." 
“There are prospects of single-agent therapy, with better tolerability and less toxicity, or potentially more impressive results with potent combination therapies. It may be possible to achieve previously unattainable goals of durable remissions, complete responses, and most importantly, improved survival benefits in triple-negative breast cancer, as well as change the stigma associated with limited targets, difficult treatment choices and dismal prognoses.” with improved outcomes. Patients who attain a 5-year disease-free benchmark have a better outcome compared with those patients who relapse early in the disease course. In this clinical setting, recurrence or progression is unlikely to occur. Because TNBC has a poor prognosis, this 5-year benchmark is a significant goal to achieve. Conventional chemotherapy remains the backbone of adjuvant systemic treatment for most patients with early TNBC. Currently, evaluation of adjuvant chemotherapy treatments in TNBC is retrospective in nature, thus, the best drugs or drug combinations have not yet been established in prospective randomized trials. Metastatic breast TNBC accounts for a significant subset of TNBC and also accounts for a considerable number of breast cancer deaths due to its highly aggressive nature. In patients with previously treated TNBC who have progressed with metastatic disease, there is no standard protocol for the first-line treatment in this setting. The prognosis of patients presenting with metastatic disease remains very poor, and the treatment goal is palliative.

Women who have the BRCA1 gene mutation are more likely to have TNBC, with suggestions being made that platinum-based chemotherapy may be more useful in $B R C A$-associated disease. It is more prevalent in non-Caucasian patients. Due to the before-mentioned lack of receptors in TNBC, extensive research has gone into looking at 'other' targeting options for this complex cancer, to improve response rates, and more importantly, overall survival $[1,2,4]$.

Antiangiogenesis drugs, such as bevacizumab (an antibody to VEGF) and treatment with PARP inhibitors have led to conflicting results in patients with TNBC. EGFR inhibitors have also been looked at but showed no real clinical benefit $[1,2]$.

Neoadjuvant bevacizumab was evaluated in TNBC in the GeparQuinto (GBG 44) study. In this study, the addition of bevacizumab, to chemotherapy, resulted in increased rates of pCR, which was the primary end point of the trial. The pCR rate was $27.9 \%$ without, and $39.3 \%$ with bevacizumab. PCR is a surrogate marker for overall survival in some schools of thought. The GBG 44 concluded that adding bevacizumab to the chemotherapy backbone in patients with TNBC, significantly increases pCR rates compared with chemotherapy without bevacizumab. These findings were not confirmed in the NSABP B-40 neoadjuvant trial in which more than 1200 patients were enrolled. Although the pCR rate in patients with TNBC receiving bevacizumab was much higher, it was not statistically or clinically significant. In contrast, the GBG 44 study examined the rate of $\mathrm{pCR}$ according to hormone receptor status. The effect of bevacizumab was more pronounced in the hormone receptor-positive subset of patients ( $15.1 \%$ without bevacizumab vs $23.2 \%$ with bevacizumab). The effect was weaker in the hormone receptor-negative subset $(47.1 \%$ without bevacizumab vs $51.5 \%$ with bevacizumab). Bevacizumab plus chemotherapy was associated with an increase in toxicity in both clinical trials, particularly hypertension, cardiac dysfunction and mucositis. There was an increased incidence of grade 3 events, often leading to treatment discontinuations. In the adjuvant setting, BEATRICE, a randomized Phase III trial, researched adjuvant bevacizumab in patients with TNBC. The addition of bevacizumab versus chemotherapy alone failed to show improvement in progression-free and overall survival $[5,6]$.

Due to their association with BRCA1, PARP inhibitors have come to light in early and advanced TNBC. There are at least ten trials, used in combination with chemotherapy, which are still under investigation. In a Phase II trial, veliparib, an oral PARP inhibitor, was shown to increase rates of pCR with carboplatin in addition to standard chemotherapy used in the subset of patients with early TNBC in the neoadjuvant setting. It will now be investigated in a Phase III trial. Other PARP inhibitors such as olaparib and niraparib have also indicated some promising results, but there is still more work to be done $[1,2,7]$.

In a further attempt to find novel targets, great interest has been shown in the androgen receptor (AR). Usually, the AR is co-expressed with ER, PR and HER2. In TNBC, AR is expressed in about $20 \%$ of these patients (at least $10 \%$ of cells need to stain positive). AR is a relatively small subset of TNBC, meaning only about $2 \%$ of overall breast cancer cases may benefit from blocking this target. It also correlates with the fact that AR-positive tumors are usually low grade, smaller tumors with low proliferation indexes, which is not characteristic of TNBCs. While it does not have a significant prognostic value, it is nonetheless undoubtedly a new target for inhibition especially due to the accessibility of AR inhibitors. An initial Phase II 
study using bicalutamide showed clinical benefit. The AR inhibitor enzalutamide, a drug used in prostate cancer, also shows promise as a single agent by allowing for some good responses. End points in studies showed improvement in clinical benefit rate and progression-free survival. A Phase II study has shown that enzalutamide is also tolerated well by patients with minimal toxicities, which is an important consideration in the palliative setting [8-10].

The era of immunotherapy makes it worthwhile to look the impact of this therapy on TNBC, an aggressive and difficult to treat disease with very limited therapeutic options. Responses to immunotherapy have been significant in other cancers, such as lung cancer and melanoma, and it is therefore hoped the prognosis of TNBC could be improved altogether [11].

The targets in TNBC related to immunotherapy are said to be due to the association of tumor-infiltrating lymphocytes (TIL). They might play a role in predictive value in end points such as disease-free intervals and overall survival most likely by aiding immune response. TILs also have prognostic value, as a better outcome is associated with their expression due to an increased susceptibility to immunotherapy [11,12].

TNBC also expresses PD-L1, a transmembrane protein in as many as $50 \%$ of cancers, and is more likely to be AR negative. Its receptor is PD-1 and it also binds PD-L2 as its other ligand. TNBC is also more likely to express the CTLA4. Both of these can possibly co-express PIK3A and PTEN, which may allow an opportunity for a dual blockade of these pathways for more efficient tumor response, but there is no evidence backing this theory yet. PD-L1 and CTLA 4 are immune checkpoints that are expressed on the surface of antigen presenting cells in the initiator and effector phase of T-cell activation, respectively. They are responsible for 'switching off' the T cell. Inhibition of these checkpoints allows for overexpression of the immune system, which is a powerful mechanism to defeat tumor cells. These checkpoints have been extensively researched and proven powerful targets in cancers such as melanoma and lung cancer [11,12].

Recently, it was shown that PD-L1 upregulation is more common in basal breast cancers and is associated with a higher T-cell cytotoxic immune response. The PD-L1 upregulation is associated with better survival and response to chemotherapy. Reactivation of inactive TILs by PD-L1-inhibitors represents a promising strategy in PD-L1 upregulated basal breast cancer [13].

PD-1 blockers in TNBC are still under investigation, namely nivolumab and pembrolizumab. Studies are investigating the drugs' effectiveness as single agents as well as in combination with other chemotherapy agents, for example, eribulin and nabpaclitaxel. Phase III trials are now underway for these drugs. They are extremely promising in a landscape of previously poor response rates in advanced/metastatic cancers as well as in inflammatory breast cancers. Also, severe toxicities are minimal and manageable. These checkpoint inhibitors are already registered in melanoma and lung cancer [7].

In initial Phase I investigation, PD-L1 blocker MPDL3280A, showed promise in patients with TNBC, who have received multiple previous therapies. Subsequently the treatment, now called atezolizumab, has shown great promise if used upfront in metastatic TNBC, in combination with nab-paclitaxel. It showed objective response rates of $66.7 \%$, regardless of PD-L1 status. Now in Phase II studies, it also showed good response rates in second and third line settings and the results are deemed to be very encouraging. Toxicity profiles were acceptable and the drugs tolerated fairly well by the patients [14].

Like PD-L1, indoleamine 2,3-dioxygenase (IDO), is an immunomodulatory enzyme also expressed on dendritic cells and tumor cells, and is also a target for antitumor immune responses. IDO, which can be induced, catalyzes the rate-limiting step in the catabolism of tryptophan. The breakdown and depletion of tryptophan causes inactivation of T cells, thus, IDO inhibition counteracts the immunosuppression. The IDO pathway inhibitor, indoximod, is also being investigated in combination with docetaxel in the Phase II setting versus docetaxel by itself in metastatic TNBC as well as in hormone receptor-positive breast cancer $[7,11,15]$.

Anticancer vaccines are recognized in the treatment of prostate cancer, where Sipuleucel-T is approved for minimal symptomatic metastatic castrate resistant prostate cancer on the basis that it showed an overall survival benefit in the IMPACT trial. It is an autologous cellular immunotherapy, where peripheral blood mononuclear cells are obtained through 
leukapheresis, activated with prostatic acid phosphatase and reinfused to mediate immune reactions against prostate cells. Similarly, in TNBC, trials are underway to use short-term cultured autologous TILs in combination with pembrolizumab. Also autologous intratumoral administration of cMET (a novel tumorassociated antigen, associated with proliferation in certain cancers), with redirected $\mathrm{T}$ cells in patients with metastatic TNBC is also under consideration. Hopefully such therapeutic vaccines will play a significant role in this disease. Other possible therapeutic vaccine targets are MAGE-A, NY-ESO and MUC-1. MAGE-A and NY-ESO (the latter is assumed to be the most immunogenic), both have a higher incidence of expression in TNBC. MAGE-A and NY-ESO are cancer-testis antigens and are also under investigation for their therapeutic value in vaccine-based treatment in TNBC. It is not known whether or not they have an effect on prognosis, but it is thought that the preoperative setting would be the best setting to test the efficacy of this vaccination, as it is so highly expressed in TNBC. MUC-1, associated with a better prognosis, is used for diagnostic purposes of certain tumors. It has also been shown to bind to p53 and prevents p53-associated apoptosis, thus preventing cell death. MUC-1 vaccines have; however, failed to show any responses [7,11,15-17].

Safety and immunogenicity testing for a personalized polypeptide DNA vaccine for metastatic TNBC is also currently underway. A pool of different peptide candidates taken from the hosts pre-existing immunity is used to select individual vaccine antigens. The vaccine is used in patients who are pretreated and failed standard chemotherapy regimens. Complete responses were seen in one or two patients, and also there is potential evidence for a survival benefit with a good safety profile. The median progression-free survival time and median overall survival time were 7.5 months and 11.5 months in the TNBC subset. The use of vaccine therapy, Ankara, is also said to be an attractive target for immunotherapy. Ankara is a $p 53$ gene product, which has the potential to induce expansion of $p 53$-specific cytotoxic T lymphocytes and to promote T-cellmediated antitumor responses. It is under investigation in TNBC in combination with PD-L1 inhibitors in patients that have developed resistance to current therapies, but it is useful in patients that test positive for the $p 53$ mutation.
There are other vaccines under investigation in advanced disease such as STEMVAC, a multiple antigen vaccine, as well as a molecule called 'oncofetal antigen'. Antitumor vaccines are still in very early stages of investigation $[7,18,19]$.

Historically, interleukin (IL) therapy was the first, but very toxic immunotherapy proven to work in cancer such as melanoma. The growth of TNBC is dependent on inflammatory signaling and production of ILs as well. An IL-1 $\beta$ receptor antagonist - Anakinra, used in the treatment of rheumatoid arthritis - is also being looked at in a pilot safety trial in combination with chemotherapy and a dendritic cell vaccine in locally advanced TNBC. Some studies show that it may work synergistically to improve cancer vaccines. Another drug, ruxolitinib, which is an IL-6/JAK/Stat pathway blocker used in myeloproliferative disorders, is also being looked at in a Phase II trial. Ruxolitinib with paclitaxel is being investigated in patients with locally advanced, inflammatory TNBC, in the preoperative setting. Patients with metastatic disease will also be eligible for this trial. Tumor vaccines still have a long way to go before they can be applied to clinical practice $[7,20]$.

There are certainly vast and exciting options on the horizon, and some already feasible immunotherapy options in TNBC: starting again with IL therapy, multiple therapeutic vaccines, checkpoint inhibition, known to show significant responses in other cancers and through to autologous cellular immunotherapy. There are prospects of single-agent therapy, with better tolerability and less toxicity, or potentially more impressive results with potent combination therapies. It may be possible to achieve previously unattainable goals of durable remissions, complete responses, and most importantly, improved survival benefits in TNBC, as well as change the stigma associated with limited targets, difficult treatment choices and dismal prognoses.

Financial \& competing interests disclosure The authors have no relevant affiliations or financial involvement with any organization or entity with a financial interest in or financial conflict with the subject matter or materials discussed in the manuscript. This includes employment, consultancies, honoraria, stock ownership or options, expert testimony, grants or patents received or pending, or royalties.

No writing assistance was utilized in the production of this manuscript. 


\section{References}

1 Hudis CA, Gianni L. Triple-negative breast cancer: an unmet medical need. Oncologist 16(Suppl. 1), 1-11 (2011).

2 Rapoport BL, Nayler S, Demetriou GS et al. Triple negative breast cancer pathologic diagnosis and current chemotherapy treatment options. Eur. Oncol. Haematol. 10(1), 35-42 (2014).

3 Cheang MC, Voduc D, Bajdik C et al. Basal-like breast cancer defined by five biomarkers has superior prognostic value than triple-negative phenotype. Clin. Cancer Res. 14(5), 1368-1376 (2008).

4 Liedtke C, Mazouni C, Hess KR et al. Response to neoadjuvant therapy and long-term survival in patients with triple-negative breast cancer. J. Clin. Oncol. 26(8), 1275-1281 (2008).

5 Cameron D, Brown J, Dent R et al. Adjuvant bevacizumab-containing therapy in triple-negative breast cancer (BEATRICE): primary results of a randomised, Phase 3 trial. Lancet Oncol. 14(10), 933-942 (2013).

6 Gerber B, Loibl S, Eidtmann $\mathrm{H}$ et al. Neoadjuvant bevacizumab and anthracyclinetaxane-based chemotherapy in 678 triplenegative primary breast cancers; results from the geparquinto study (GBG 44). Ann. Oncol. 24(12), 2978-2984 (2013).

7 Breast Cancer Trials. www.breastcancertrials.org
8 Safarpour D, Pakneshan S, Tavassoli FA et al. Androgen receptor (AR) expression in 400 breast carcinomas: is routine $\mathrm{AR}$ assessment justified? Am. J. Cancer Res. 4(4), 353-368 (2014).

9 Gucalp A, Tolaney S, Isakoff SJ et al. Phase II trial of bicalutamide in patients with androgen receptor-positive, estrogen receptor-negative metastatic breast cancer. Clin. Cancer Res. 19(19), 5505-5512 (2013).

10 Traina T, Miller K, Yardley A et al. Results from a Phase 2 study of enzalutamide (ENZA), an androgen receptor (AR) inhibitor, in advanced $\mathrm{AR}^{+}$triple-negative breast cancer (TNBC). J. Clin. Oncol. 33(Suppl.), Abstract 1003 (2015).

11 Stagg J, Allard B. Immunotherapeutic approaches in triple-negative breast cancer: latest research and clinical prospects. Ther. Adv. Med. Oncol. 5(3), 169-181 (2013).

12 Adams S, Goldstein LJ, Sparano JA et al. Tumor infiltrating lymphocytes (TILs) improve prognosis in patients with triple negative breast cancer (TNBC). Oncoimmunology 4(9), e985930 (2015).

13 Sabatier R, Finetti P, Mamessier E et al. Prognostic and predictive value of PDL1 expression in breast cancer. Oncotarget 6(7), 5449-5464 (2015).

14 Goodman A. Anti-PD-L1 agent shows activity in early study of triple-negative breast cancer. Presented at: The ASCO POST. 6(10), 10 June 2015. www.ascopost.com

15 Soliman H, Mediavilla-Varela M, Antonia S. Indoleamine 2,3-dioxygenase: is it an immune suppressor? Cancer J. 6(4), 354-359 (2010).

16 Kantoff PW, Higano CS, Shore ND. Sipuleucel-T immunotherapy for castrationresistant prostate cancer. N. Engl. J. Med. 363(5), 411-422 (2010).

17 Curigliano G, Viale G, Ghioni M et al. Cancer-testis antigen expression in triple-negative breast cancer. Ann. Oncol. 22(1), 98-103 (2011)

18 Takahashi R, Toh U, Iwakuma $\mathrm{N}$ et al. Feasibility study of personalized peptide vaccination for metastatic recurrent triple-negative breast cancer patients. Breast Cancer Res. 16(4), R70 (2014).

19 McShane H, Pathan AA, Sander CR et al. Recombinant modified vaccinia virus Ankara expressing antigen 85A boosts BCG-primed and naturally acquired antimycobacterial immunity in humans. Nat. Med. 10(11), 1240-1244 (2004).

20 Hartman ZC, Poage GM, den Hollander P et al. Growth of triple-negative breast cancer cells relies upon coordinate autocrine expression of the proinflammatory cytokines IL-6 and IL-8. Cancer Res. 73(11), 3470-3480 (2013). 